\title{
Ipilimumab-Induced Enteritis without Colitis: A New Challenge
}

\author{
Marcus Messmer ${ }^{a} \quad$ Sunita Upreti ${ }^{b} \quad$ Yaman Tarabishy $^{c}$ \\ Nikhilesh Mazumder $^{\mathrm{a}}$ Reezwana Chowdhury $^{\mathrm{d}}$ Mark Yarchoan $^{\mathrm{e}}$ \\ Matthias Holdhoff \\ ${ }^{a}$ Department of Medicine, Johns Hopkins University School of Medicine, \\ Baltimore, MD, USA; ${ }^{b}$ Department of Medicine, Drexel University College of Medicine, \\ Philadelphia, PA, USA; 'Department of Pathology, Johns Hopkins University School of \\ Medicine, Baltimore, MD, USA; ${ }^{d}$ Division of Gastroenterology, Department of Medicine, \\ Johns Hopkins University School of Medicine, Baltimore, MD, USA; ${ }^{\mathrm{e}}$ The Sidney Kimmel \\ Comprehensive Cancer Center at Johns Hopkins, Johns Hopkins University School of \\ Medicine, Baltimore, MD, USA
}

\section{Keywords}

Ipilimumab · CTLA4 inhibitor · Enteritis · Colitis · Immunotherapy · Melanoma

\begin{abstract}
Introduction: Ipilimumab is an immune checkpoint inhibitor targeting cytotoxic Tlymphocyte associated antigen 4 (CTLA4), approved to treat metastatic melanoma. It was the first therapy shown to prolong survival in a large, randomized clinical trial. However, immune-related adverse events are common and can be severe. Enterocolitis is a common adverse event with ipilimumab, but enteritis without colitis has not been previously described. Case Report: An 83-year-old man presented to our hospital with grade 3 diarrhea for 5 days. One month prior, he had started treatment with ipilimumab for metastatic melanoma. On presentation, he was found to have severe electrolyte disturbances, including hyponatremia, hypokalemia, and acute kidney injury. Causes of infectious diarrhea were excluded, and he was treated with corticosteroids for presumed ipilimumab-associated enterocolitis. However, colonoscopy revealed normal mucosa, both grossly and on pathology of random biopsies. Steroids were weaned but his symptoms recurred. He then underwent upper endoscopy with enteroscopy. Biopsy of the duodenum was notable for acute inflammation, villous blunting, and other changes consistent with ipilimumab-associated injury. He was restarted on high-
\end{abstract}


dose steroids and his symptoms resolved. Discussion: Ipilimumab-induced enteritis is a serious and potentially life-threatening immune related adverse event that warrants prompt recognition and aggressive management. As in cases of ipilimumab-associated enterocolitis, steroids are an effective therapy. Enteritis without colitis should be suspected in patients on ipilimumab who present with severe diarrhea but have a normal colonoscopy.

(C) 2016 The Author(s)

Published by S. Karger AG, Basel

\section{Introduction}

In the last few years, the clinical development of novel immune checkpoint inhibitors has revolutionized the treatment of metastatic melanoma. In 2010, the immune checkpoint inhibitor ipilimumab was the first therapy to ever demonstrate a clear survival benefit in melanoma in a large randomized clinical trial [1]. Ipilimumab is an inhibitor of cytotoxic Tlymphocyte associated antigen 4 (CTLA4). CTLA4 activation induces intracellular pathways that downregulate T-cell activation. By inhibiting CTLA4, ipilimumab results in prolonged activation of T lymphocytes, leading to T-cell mediated activity against tumor cells [2]. More recently, the treatment of metastatic melanoma has benefited from the approval of two additional immune checkpoint inhibitors, nivolumab [3] and pembrolizumab [4], which both inhibit the immune checkpoint programmed cell death protein 1 (PD-1). Although treatment with these novel therapies can provide durable responses in many patients, instances of immune-related adverse events (irAEs) linked to immune activation in nontarget tissues are sometimes observed. In patients treated with ipilimumab, the most common AEs are enterocolitis, hepatitis, dermatitis, neuropathy, and endocrinopathies [1]. Enterocolitis is both common and debilitating, with $7 \%$ of patients developing severe, life-threatening, or fatal effects, and 1\% resulting in intestinal perforation [1]. Despite enterocolitis being commonly observed with ipilimumab, with detailed guidelines on observation and management, severe enteritis without colitis has not, to our knowledge, been previously described.

\section{Case Report}

An 83-year-old male receiving ipilimumab for metastatic melanoma presented to our hospital with grade 3 diarrhea for 5 days. He had previously been treated with pembrolizumab for 4 months, which was stopped 2 months prior to onset of his symptoms due to disease progression. He was initiated on ipilimumab therapy 1 month before his presentation to our hospital. He noted several days of watery, non-bloody diarrhea and crampy abdominal pain. He had no recent travel and had not been exposed to anyone with similar complaints. He presented to an outside emergency room, where he had an unremarkable CT scan of the abdomen and was treated empirically with fluids, hydrocortisone $100 \mathrm{mg}$, and metronidazole. He was discharged home but continued to have symptoms and returned to the emergency department. He was again treated with IV fluids, and transferred to our hospital. On presentation, he was well appearing, afebrile, without tachycardia or hypotension. His abdomen was benign, with no tenderness or distension and normal bowel sounds. Labs were notable for hyponatremia, acute kidney injury, severe gap and non-anion gap metabolic acidosis, hypokalemia, hypocalcemia, and hypomagnesemia. White blood cell count was elevated to $11.25 \mathrm{~K} / \mathrm{cu} \mathrm{mm}$ with $30 \%$ bands. Urinalysis revealed moderate ketones. Stool studies were negative for clostridium difficile toxin B by PCR, EHEC-EIA, bacterial culture, 
giardia, ova and parasites, microsporidium, and cryptosporidium. His stool was positive for fecal lactoferrin. Interpretation of the outside hospital CT abdomen with IV contrast was performed by our radiology department. This was significant for minimal thickening of the terminal ileum, with few prominent adjacent lymph nodes. He was started on IV methylprednisolone $125 \mathrm{mg}$ twice a day for presumed ipilimumab-induced colitis. His diarrhea began to improve and he underwent a colonoscopy, which showed no erythema, ulceration, or other mucosal abnormality (Fig. 1). Random colon biopsies also showed normal mucosa (Fig. 2). With colitis ruled out by colonoscopy, steroids were tapered to prednisone 60 mg daily, but he had worsening of his diarrhea and electrolyte abnormalities. At this point, prednisone doses were increased to $80 \mathrm{mg}$ twice daily $(1 \mathrm{mg} / \mathrm{kg})$ and an upper endoscopy with push enteroscopy was performed. There was a small duodenal ulcer but the remainder of the duodenum and jejunum had normal appearing mucosa (Fig. 3). Random biopsies of the small bowel were taken, and pathology was notable for duodenal mucosa with acute inflammation, lymphoplasmacytic expansion of the lamina propria, villous blunting, and increased apoptotic bodies (Fig. 4). This pathology is consistent with ipilimumab-associated injury. The patient continued to improve and was discharged home on a 4-week steroid taper. After reaching a dose of hydrocortisone $30 \mathrm{mg}$ daily, he developed bloody diarrhea, fatigue, and hypotension, requiring hospital admission. He underwent sigmoidoscopy, which showed normal mucosa but random biopsies were taken and pathology was consistent with ipilimumab-associated colitis. His steroids were increased to $1 \mathrm{mg} / \mathrm{kg}$ b.i.d. and he received a dose of infliximab. His diarrhea did not recur.

\section{Discussion}

Ipilimumab-induced enteritis is a serious and potentially life-threatening immune related $\mathrm{AE}$ that warrants prompt recognition and aggressive management. Ipilimumab treatment gives rise to altered levels of antibodies to enteric flora and increased levels of fecal calprotectin, although it was not proven a reliable predictor of onset of enterocolitis [5]. Guidelines for management of enterocolitis indicate that reactions typically occur 1-2 months after initiation of the drug and often occur while the therapy is ongoing, but toxicity weeks to months after discontinuation has been observed. The FDA recommends monitoring for diarrhea, fever, hematochezia, ileus, or peritonitis with ipilimumab therapy. If severe symptoms develop, ipilimumab should be discontinued and corticosteroids started at $1-2 \mathrm{mg} / \mathrm{kg} /$ day and tapered over 1 month. Infectious etiologies should be excluded and endoscopic evaluation considered [6]. Endoscopic evaluation is warranted in patients with moderate to severe (grade $>2$ ) diarrhea and biopsies should be obtained [7]. Infliximab has been studied and proven to be effective in refractory cases [2]. This case demonstrates that ipilimumab can induce immune-mediated destruction of small bowel mucosa in the absence of or prior to large bowel involvement. This can result in severe electrolyte disturbances and hypovolemia, leading to significant morbidity. Furthermore, enteritis may precede colitis in cases of ipilimumab-associated enterocolitis. Because of the initial absence of colonic involvement, lower endoscopy will be non-diagnostic and the etiology may remain unrecognized. In severe cases of diarrhea after initiation of ipilimumab, examination of the small bowel with enteroscopy may be warranted to make the diagnosis.

In conclusion, we present a case of ipilimumab-induced enteritis weeks prior to the development of colitis. This complication should be suspected in patients with ipilimumabassociated diarrhea and a normal colonoscopy. 


\section{Acknowledgement}

The study was supported by the Sidney Kimmel Comprehensive Cancer Center core grant, P30CA006973.

\section{Statement of Ethics}

The authors have no ethical conflicts to disclose.

\section{Disclosure Statement}

The authors declare that they have no relevant conflicts of interest.

\section{References}

1 Hodi FS, O'Day SJ, McDermott DF, et al: Improved survival with ipilimumab in patients with metastatic melanoma. N Engl J Med 2010;363:711-723.

2 Pages C, Gornet JM, Monsel G, et al: Ipilimumab-induced acute severe colitis treated by infliximab. Melanoma Res 2013;23:227-230.

-3 Wolchok JD, Kluger H, Callahan MK, et al: Nivolumab plus ipilimumab in advanced melanoma. N Engl J Med 2013;369:122-133.

4 Robert C, Schachter J, Long GV, et al: Pembrolizumab versus ipilimumab in advanced melanoma. N Engl J Med 2015;372:2521-2532.

5 Quirk SK, Shure AK, Agrawal DK, et al: Immune-mediated adverse events of anticytotoxic T lymphocyteassociated antigen 4 antibody therapy in metastatic melanoma. Transl Res 2015;166:412-424.

6 Ipilimumab (package insert). Bristol-Myers Squibb Company. Princeton, 2011.

7 Fecher LA, Agrawala SS, Hodi FS, et al: Ipilimumab and its toxicities: a multidisciplinary approach. Oncologist 2013;18:733-743. 


\section{Case Reports in Oncology}

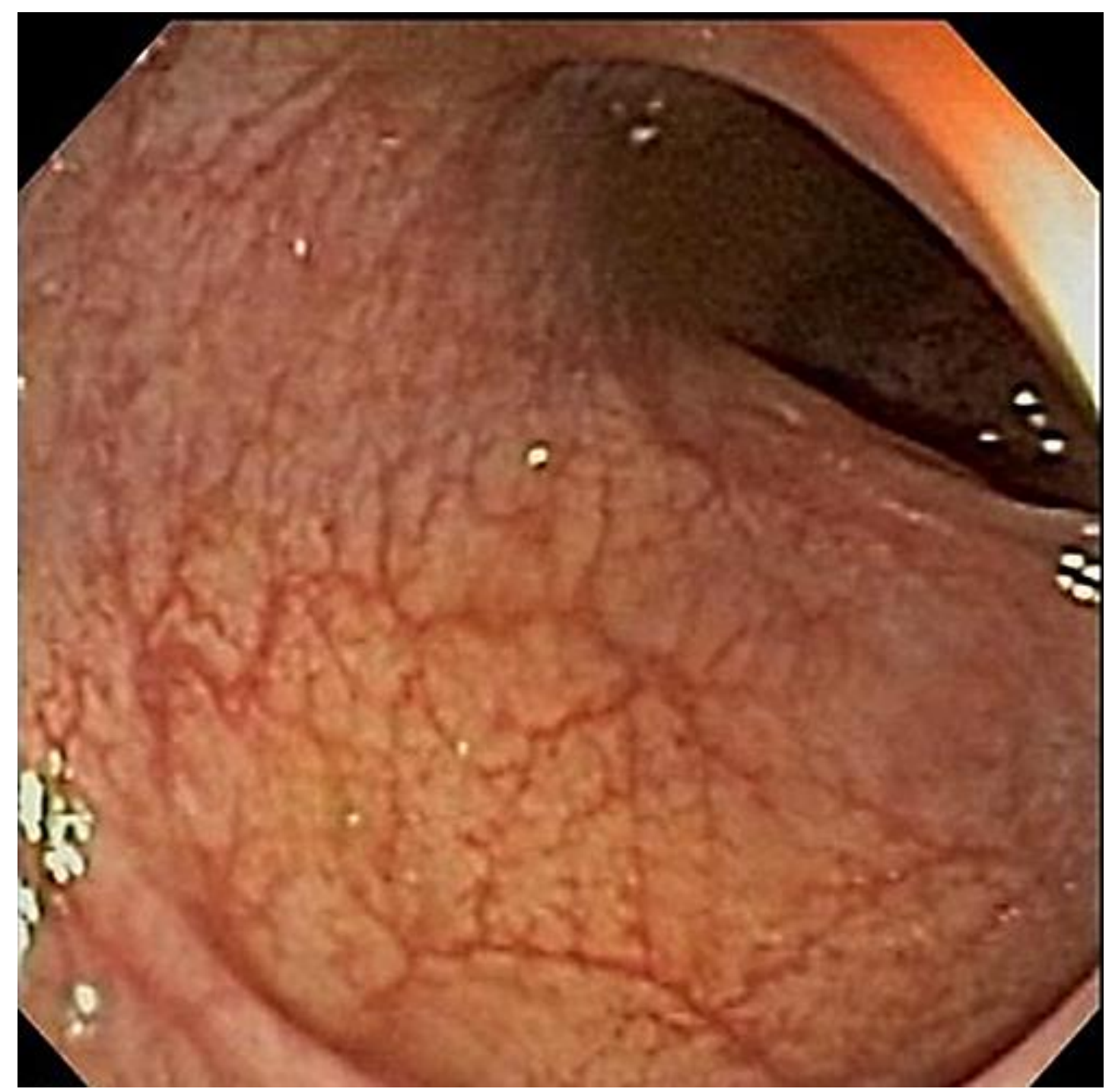

Fig. 1. Colonoscopy was performed, which revealed grossly normal mucosa. 


\section{Case Reports in Oncology}

Case Rep Oncol 2016;9:705-713

DOI: 10.1159/000452403

Messmer et al.: Ipilimumab-Induced Enteritis without Colitis: A New Challenge

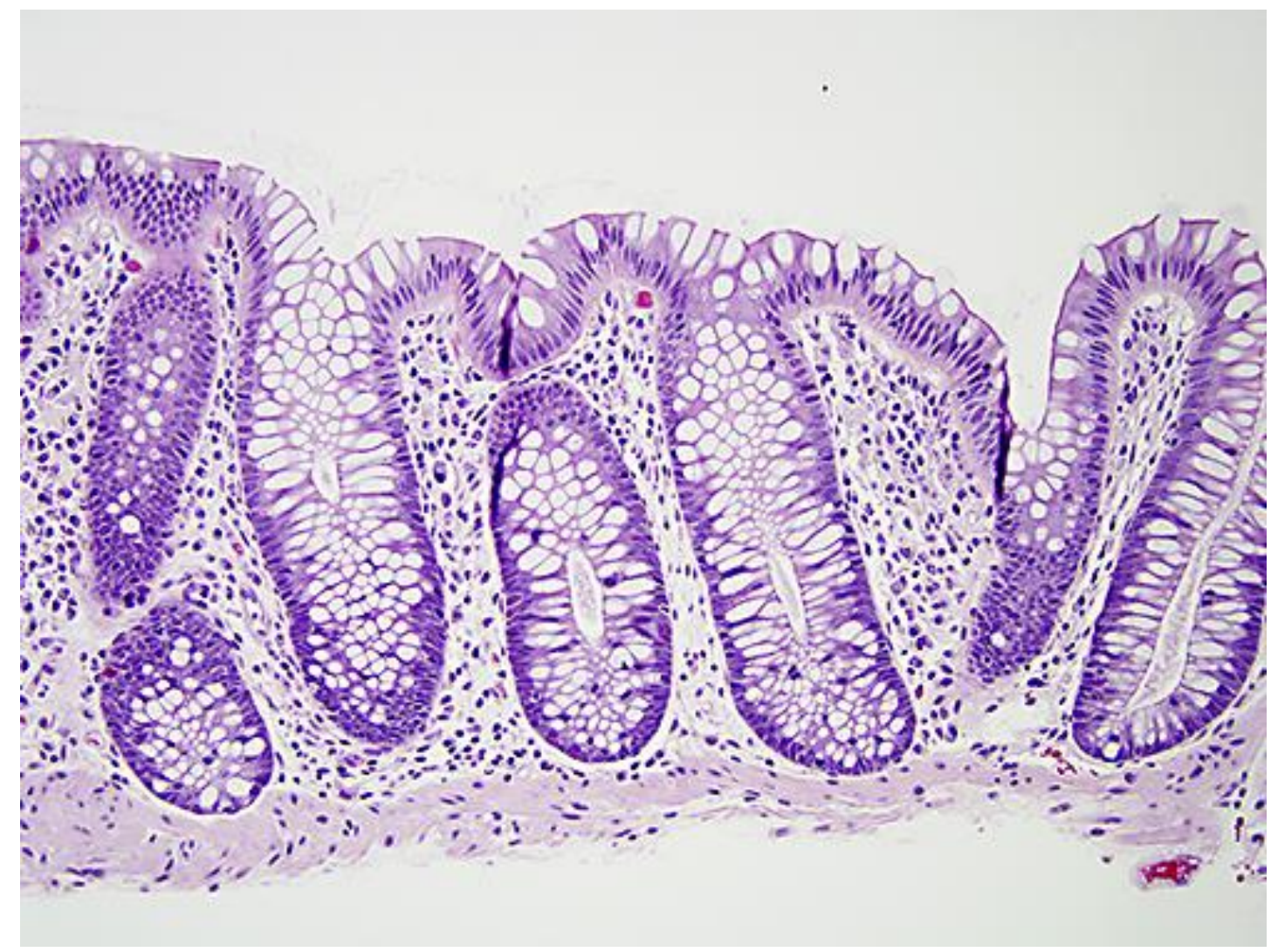

Fig. 2. Random biopsies of the colon showed normal mucosa. 


\section{Case Reports in Oncology}

\begin{tabular}{l|l}
\hline Case Rep Oncol 2016;9:705-713 \\
\hline DOI: 10.1159/000452403 & $\begin{array}{l}\text { ○ 2016 The Author(s). Published by S. Karger AG, Basel } \\
\text { www.karger.com/cro }\end{array}$ \\
\hline Messmer et al.: Ipilimumab-Induced Enteritis without Colitis: A New Challenge
\end{tabular}

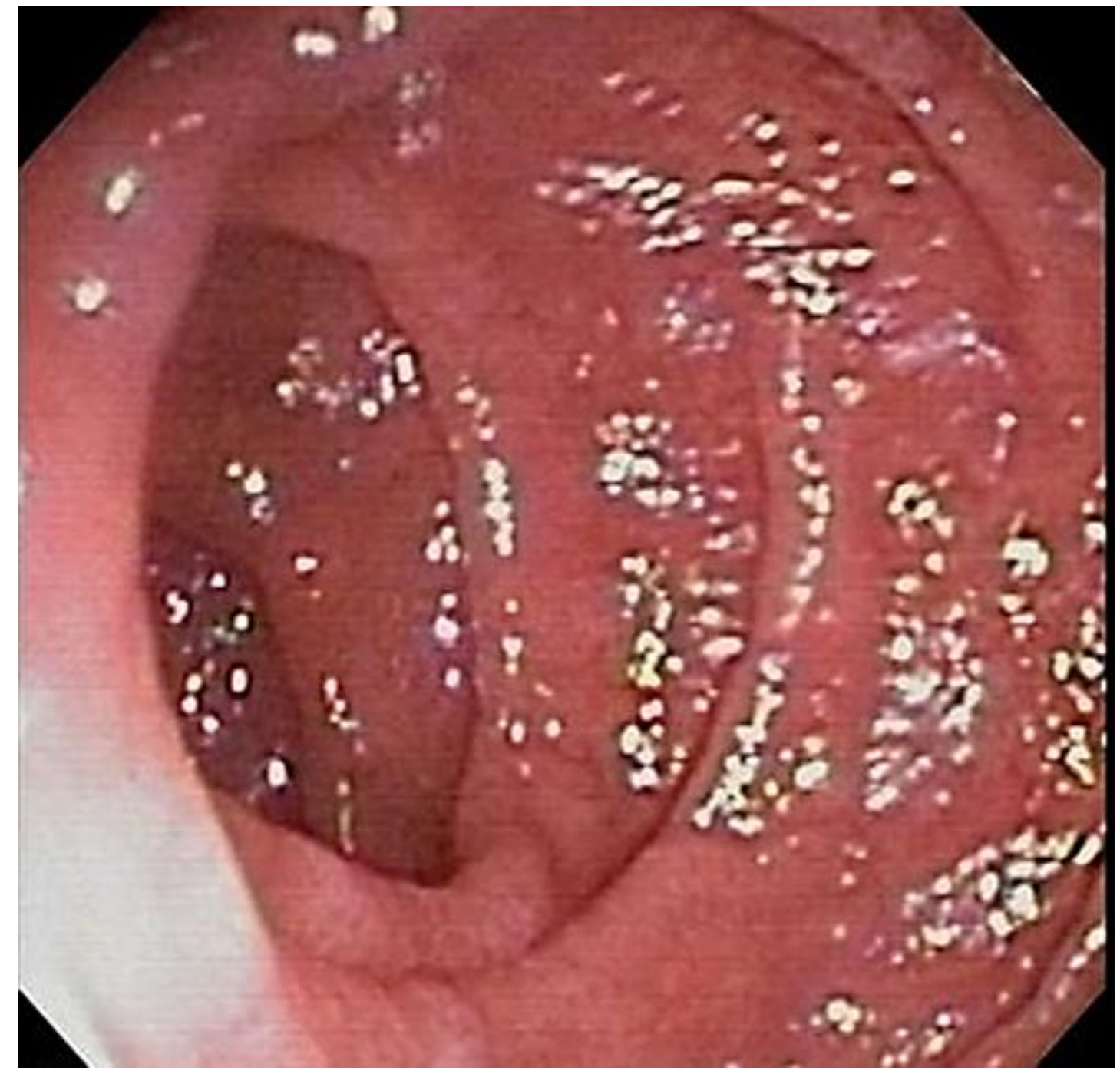

Fig. 3. On enteroscopy, the small bowel appeared grossly unremarkable. 


\section{Case Reports in Oncology}

a
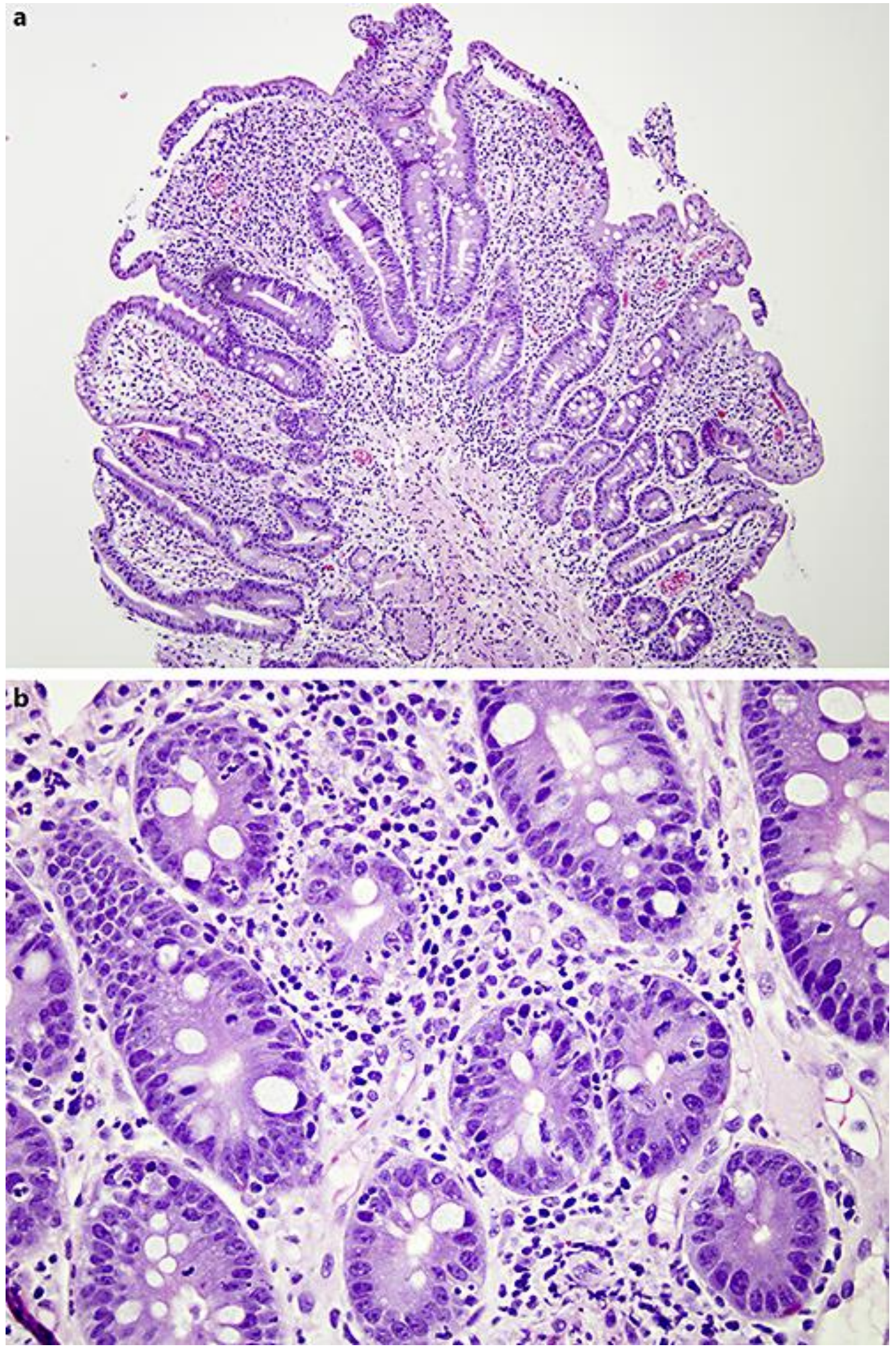
Fig. 4. Random biopsies of the duodenum were taken, which revealed villous blunting (a), as well as acute inflammation, expansion of the lamina propria, and increased apoptotic bodies (b). 